\title{
(Higgs) vacuum decay during inflation
}

\author{
Aris Joti, ${ }^{a}$ Aris Katsis, ${ }^{a}$ Dimitris Loupas, ${ }^{a}$ Alberto Salvio, ${ }^{b}$ Alessandro Strumia,,${ }^{b, c}$ \\ Nikolaos Tetradis $^{a}$ and Alfredo Urbano ${ }^{b}$ \\ ${ }^{a}$ Department of Physics, National and Kapodistrian University of Athens, \\ Zographou 15784 , Greece \\ ${ }^{b}$ CERN, Theoretical Physics Department, \\ Geneva, Switzerland \\ ${ }^{c}$ Dipartimento di Fisica, Università di Pisa and INFN, Sezione di Pisa, \\ Largo Bruno Pontecorvo 3, Pisa, Italy \\ E-mail: apjoti@gmail.com, Aris_Katsis@hotmail.com, \\ dloupas@physics.auth.gr, alberto.salvio@cern.ch, astrumia@cern.ch, \\ ntetrad@phys.uoa.gr, alfredo.leonardo.urbano@cern.ch
}

ABSTRACT: We develop the formalism for computing gravitational corrections to vacuum decay from de Sitter space as a sub-Planckian perturbative expansion. Non-minimal coupling to gravity can be encoded in an effective potential. The Coleman bounce continuously deforms into the Hawking-Moss bounce, until they coincide for a critical value of the Hubble constant. As an application, we reconsider the decay of the electroweak Higgs vacuum during inflation. Our vacuum decay computation reproduces and improves bounds on the maximal inflationary Hubble scale previously computed through statistical techniques.

Keywords: Higgs Physics, Solitons Monopoles and Instantons, Renormalization Group, Classical Theories of Gravity

ArXIV EPRINT: 1706.00792 


\section{Contents}

1 Introduction 1

2 General theory and sub-Planckian approximation $\quad 2$

2.1 The Hawking-Moss bounce 5

2.2 Sub-Planckian approximation to the bounce 5

$\begin{array}{llr}3 & \text { Renormalizable potential } & 8\end{array}$

3.1 Zeroth order in $H, M \ll M_{\mathrm{Pl}}$

3.2 First order in $H, M \ll M_{\mathrm{Pl}} \quad 10$

3.3 Effect of non-minimal couplings 11

4 Standard Model vacuum decay during inflation $\quad 12$

$\begin{array}{lll}\text { 4.1 SM vacuum decay for small } H & 13\end{array}$

$\begin{array}{lll}4.2 & \mathrm{SM} \text { vacuum decay for large } H \gg h_{\mathrm{cr}} & 13\end{array}$

$\begin{array}{lll}4.3 & \text { SM vacuum decay for } H \sim h_{\text {cr }} & 15\end{array}$

$\begin{array}{lll}5 & \text { Conclusions } & 17\end{array}$

\section{Introduction}

A false vacuum can decay through quantum tunnelling that leads to nucleation of regions of true vacuum. The rate of this non-perturbative phenomenon is exponentially suppressed by the action of the 'bounce', the field configuration that dominates the transition [1].

Coleman and de Luccia showed how to account for gravitational effects [2]. Their formalism can be simplified by restricting to sub-Planckian energies, which are the only ones for which Einstein gravity can be trusted. Simplified expressions were obtained in [3, 4] for the decay of flat space-time.

We extend here the simplified formalism to tunnelling from de Sitter space (positive energy density), which is relevant during inflation with Hubble constant $H$. It is known that gravitational effects can dramatically enhance the tunnelling rate. The qualitative intuition is that a de Sitter space has a Gibbons-Hawking 'temperature' $T=H / 2 \pi[5]$ that gives extra 'thermal' fluctuations that facilitate tunnelling. Equivalently, light scalar fields $h$ undergo fluctuations $\delta h \sim H / 2 \pi$ per $e$-folding.

We show that tunnelling from de Sitter can be described by a simplified formalism assuming that $H$ and the bounce energy density are sub-Planckian. We also show how to include perturbatively the small extra Planck-suppressed corrections due to gravity.

Vacuum decay in the presence of gravity receives an extra contribution from another bounce, known as the Hawking-Moss (HM) solution: a constant field configuration that sits at the top of the potential barrier [6]. Being constant, it has the higher $\mathrm{O}(5)$ symmetry of 4-dimensional de Sitter space, while the Coleman-de Luccia (CdL) bounce is only $\mathrm{O}(4)$ 
symmetric. The Hawking-Moss contribution to vacuum decay vanishes for flat space, which corresponds to $H=0$. We show that the Coleman bounce continuously deforms into the Hawking-Moss bounce, and the two become equal at a critical value of $H$, usually equal to $1 / 2$ of the curvature of the potential at its maximum.

Furthermore, the simplified formalism allows to easily include perturbatively the effect of a general non-minimal scalar coupling to gravity, as a modification of the effective scalar potential.

The above features are relevant for the possible destabilization of the Standard Model (SM) vacuum during inflation. The Higgs field can fluctuate towards values a few orders of magnitude below the Planck scale, for which its potential can be deeper than for the electroweak vacuum [7-23]. If vacuum decay happens during inflation, the regions of true vacuum expand and engulf the whole space $[2,18,24]$. This catastrophic scenario is avoided if $H$ is small enough that vacuum decay is negligibly slow. The derivation of a precise bound on the scale of inflation has been based mainly on a stochastic approach, relying on the numerical solution of Fokker-Planck or Langevin equations that describe the real-time evolution of fluctuations in the scalar field. We pursue here the alternative approach of computing the Euclidean tunnelling rate. We reproduce some previous results and correct others.

The paper is structured as follows. In section 2 we derive a simple approximation for sub-Planckian vacuum decay. In section 3 we validate the analytical expressions by focusing on a toy renormalizable scalar potential, and show how the Coleman bounce connects to the Hawking-Moss bounce. In the final section 4 we obtain bounds on the scale of inflation $H$ by computing the tunnelling rate, dominated by the Hawking-Moss bounce. Conclusions are given in section 5 .

\section{General theory and sub-Planckian approximation}

Coleman and de Luccia [2] developed the formalism for computing vacuum decay from a de Sitter space with Hubble constant $H$ taking gravity into account. In this section we review this formalism, extend it to a general non-minimal coupling of the Higgs to gravity and then derive simplified expressions that hold in the sub-Planckian limit $H, M \ll M_{\mathrm{Pl}}$, where $M$ is the mass scale that characterises the scalar potential and thereby the bounce. Within Einstein gravity this approximation applies to all cases of interest: indeed, Einstein gravity is non-renormalizable and must be replaced by some more fundamental theory at the Planck scale or below it. Furthermore, the Hubble constant during inflation must be sub-Planckian to reproduce the smallness of the inflationary tensor-to-scalar ratio.

Coleman and de Luccia assumed that the space-time probability density of vacuum decay is exponentially suppressed by the action of a 'bounce' configuration, like in flat space. ${ }^{1}$ We consider the Euclidean action of a scalar field $h(x)$ in the presence of gravity,

$$
S=\int d^{4} x \sqrt{\operatorname{det} g}\left[\frac{1}{2}(\nabla h)^{2}+V(h)-\frac{\mathcal{R}}{16 \pi G}-\frac{f(h)}{2} \mathcal{R}\right],
$$

\footnotetext{
${ }^{1}$ The proof valid in flat space cannot be extended to de Sitter space because the Hamiltonian is not well defined. One cannot isolate the ground state by computing a transition amplitude in the limit of infinite time, because Euclidean de Sitter has finite volume, so that multi-bounce configurations cannot be resummed in a dilute gas approximation.
} 
where $G \equiv 1 / M_{\mathrm{Pl}}^{2} \equiv 1 /\left(8 \pi \bar{M}_{\mathrm{Pl}}^{2}\right) \equiv \kappa / 8 \pi$ is the Newton constant, with $\bar{M}_{\mathrm{Pl}} \approx 2.43 \times$ $10^{18} \mathrm{GeV}$. For the time being $V$ and $f$ are generic functions of the scalar field $h$. The action in eq. (2.1) is the most general action for the metric $g_{\mu \nu}$ and $h$ up to two-derivative terms: an extra generic function $Z(h)$ multiplying the kinetic term of the scalar field can be removed by redefining $h$. The classical equations of motion for gravity and for $h$ are [25]

$$
\begin{aligned}
\left(\bar{M}_{\mathrm{Pl}}^{2}+f\right)\left(\mathcal{R}_{\mu \nu}-\frac{g_{\mu \nu}}{2} \mathcal{R}\right) & =\nabla_{\mu} h \nabla_{\nu} h-g_{\mu \nu}\left[\frac{(\nabla h)^{2}}{2}+V+\nabla^{2} f\right]+\nabla_{\mu} \nabla_{\nu} f, \\
\nabla^{2} h+\frac{1}{2} \frac{d f(h)}{d h} \mathcal{R} & =\frac{d V(h)}{d h},
\end{aligned}
$$

where $\nabla^{2}=\nabla_{\mu} \nabla^{\mu}$. The use of these equations allows us to simplify the action. Taking the trace of eq. (2.2) one finds

$$
\left(\bar{M}_{\mathrm{Pl}}^{2}+f(h)\right) \mathcal{R}=(\nabla h)^{2}+4 V(h)+3 \nabla^{2} f(h) .
$$

Substitution in eq. (2.1) gives

$$
S=-\int d^{4} x \sqrt{\operatorname{det} g}\left[V(h)+\frac{3}{2} \nabla^{2} f(h)\right] .
$$

The second term in the above expression is reduced to a boundary term upon integration. For the problem at hand this term vanishes and one obtains

$$
S=-\int d^{4} x \sqrt{\operatorname{det} g} V(h)
$$

We are interested in the possible decay of the false vacuum during a period in which the vacuum energy is dominated by a cosmological constant $V_{0}$. We assume that $V(h)$ has two minima, a false vacuum at $h=h_{\text {false }}$ and the true vacuum at $h=h_{\text {true }}$, with $h_{\text {true }}>h_{\text {false }}$. In the following we will set $h_{\text {false }}=0$ without loss of generality. The two minima are separated by a maximum of the potential. We identify $V_{0}=V(0)$ and split the potential as

$$
V(h)=V_{0}+\delta V(h)
$$

such that $\delta V(0)=0$. The vacuum energy density $V_{0}$ induces a de Sitter space with curvature $\mathcal{R}=12 H^{2}$, where the Hubble rate $H$ is given by

$$
H^{2}=\frac{V_{0}}{3 \bar{M}_{\mathrm{Pl}}^{2}}
$$

(we assume without loss of generality $f(0)=0$ : a non-vanishing value of $f(0)$ can be absorbed in a redefinition of $\kappa$ ). In the following we will collectively denote with $\Phi_{\text {false }}$ the field configuration with this de Sitter background and $h=0$. In the semiclassical (small $\hbar$ ) limit the decay rate $\Gamma$ of the false vacuum per unit of space-time volume $\mathcal{V}$ is given by $[1,2,26,27]$

$$
\frac{d \Gamma}{d \mathcal{V}}=A e^{-\mathcal{S} / \hbar}(1+\mathcal{O}(\hbar))
$$


where $A$ is a quantity of order $M^{4}$, where $M$ is the mass scale in the potential. The dominant effect is the bounce action $\mathcal{S}$. It is given by

$$
\mathcal{S}=S\left(\Phi_{B}\right)-S\left(\Phi_{\text {false }}\right),
$$

where $\Phi_{B}$ is an unstable 'bounce' solution of the Euclidean equations of motion, such that $\mathcal{S}$ is finite and there is no other configuration with the same properties and lower $\mathcal{S}$. In the rest of the paper we set the units such that $\hbar=1$. In order to find $\Phi_{B}$ we follow [2] and introduce an $\mathrm{O}(4)$-symmetric Euclidean ansatz for the Higgs field $h(r)$ and for the geometry

$$
d s^{2}=d r^{2}+\rho(r)^{2} d \Omega^{2},
$$

where $d \Omega$ is the volume element of the unit 3-sphere. On this background, the action becomes

$$
S=2 \pi^{2} \int d r \rho^{3}\left[\left(\frac{h^{\prime 2}}{2}+V(h)\right)-\frac{\mathcal{R}}{2 \kappa}-\frac{\mathcal{R}}{2} f(h)\right],
$$

where the curvature is

$$
\mathcal{R}=-\frac{6}{\rho^{3}}\left(\rho^{2} \rho^{\prime \prime}+\rho \rho^{\prime 2}-\rho\right)
$$

and a prime denotes $d / d r$. The simplified action of eq. (2.6) becomes:

$$
S=-2 \pi^{2} \int d r \rho^{3} V(h) \text {. }
$$

The equations of motion are

$$
\begin{aligned}
h^{\prime \prime}+3 \frac{\rho^{\prime}}{\rho} h^{\prime} & =\frac{d V(h)}{d h}-\frac{1}{2} \frac{d f(h)}{d h} \mathcal{R} \\
\rho^{\prime 2} & =1+\frac{\kappa \rho^{2}}{3(1+\kappa f(h))}\left(\frac{1}{2} h^{\prime 2}-V(h)-3 \frac{\rho^{\prime}}{\rho} \frac{d f(h)}{d h} h^{\prime}\right) .
\end{aligned}
$$

Let us discuss the boundary conditions. Since in the false vacuum the space is a 4 -sphere and topology cannot be changed dynamically, the space described by $\rho$ will have the same topology; thus $\rho$ will have two zeros. One can be conventionally chosen to occur at $r=0$, and the other one at some value of $r$ that we call $r_{\max }$,

$$
\rho(0)=\rho\left(r_{\max }\right)=0 .
$$

The whole space is covered by the coordinate interval $\left[0, r_{\max }\right]$. In the de Sitter case one has $r_{\max }=\pi / H$. The equation of motion of $h$ in (2.15) and the regularity of $h$ at $r=0$ and $r=r_{\max }$ imply

$$
h^{\prime}(0)=h^{\prime}\left(r_{\max }\right)=0 .
$$

In the limit of small $H$ (i.e. large $\left.r_{\max }\right)$, the boundary condition $h^{\prime}\left(r_{\max }\right)=0$ implies $h(\infty)=0$ in view of the large volume outside the core of the bounce. Generically, in a nontrivial ( $r$-dependent) bounce $h(r)$ does not tend to the false vacuum solution, $h_{\text {false }}=0$, as $r \rightarrow r_{\max }$, unless we are in the flat space case, $r_{\max } \rightarrow \infty .^{2}$

\footnotetext{
${ }^{2}$ This can be shown whenever $h$ and $\rho$ are regular functions at $r=r_{\max }$ : by Taylor-expanding the equations of motion eqs. (2.15)-(2.16) around $r=r_{\max }$, using $h(r)=\sum_{n=1}^{\infty} c_{n}\left(r-r_{\mathrm{rmax}}\right)^{n}$ and $\rho(r)=\sum_{n=1}^{\infty} a_{n}\left(r-r_{\max }\right)^{n}:$ one obtains a set of algebraic equations that force $c_{n}=0$. Namely, for $r_{\max }<\infty$, the only regular function that goes indefinitely close to the false vacuum solution as $r \rightarrow r_{\max }$ is the false vacuum solution itself.
} 


\subsection{The Hawking-Moss bounce}

The Hawking-Moss configuration [6], which we denote with $\Phi_{\mathrm{HM}}$, is a simple unstable finite-action solution satisfying the equations of motion and boundary conditions above. In this configuration the scalar sits at the constant value $h=h_{\max }$ that maximizes ${ }^{3}$

$$
V_{H}(h) \equiv V(h)-6 H\left(h_{\max }\right)^{2} f(h),
$$

where $H(h)$ is the Hubble constant given by

$$
H^{2}(h)=\frac{\kappa V(h)}{3(1+\kappa f(h))} .
$$

The Hawking-Moss solution exists whenever $V_{H}$ has a maximum. When $f=0, h_{\max }$ coincides with the maximum of the potential. We can compute the tunnelling rate by using the simplified action in eq. (2.14), obtaining for the Hawking-Moss solution

$$
\mathcal{S}_{\mathrm{HM}}=S\left(\Phi_{\mathrm{HM}}\right)-S\left(\Phi_{\text {false }}\right)=24 \pi^{2} \bar{M}_{\mathrm{Pl}}^{4}\left[\frac{1}{V_{0}}-\frac{\left(1+\kappa f\left(h_{\mathrm{max}}\right)\right)^{2}}{V\left(h_{\max }\right)}\right] .
$$

Defining $V_{H}(h)=V_{0}+\delta V_{H}(h)$, in the limit $\delta V\left(h_{\max }\right) \ll V_{0}$ and at leading order in $\kappa$ this formula simplifies to

$$
\mathcal{S}_{\mathrm{HM}} \simeq \frac{8 \pi^{2}}{3} \frac{\delta V_{H}\left(h_{\max }\right)}{H^{4}}
$$

where $H$ can be evaluated at $h=0$. We shall examine the role of the Hawking-Moss solution for vacuum decay, finding that it is relevant for large values of $H$.

Generically, there are also non-trivial solutions with a non-constant Higgs profile $h(r)$, which, in the flat-space limit, reduce to the Coleman bounce [1]. In order to determine the various bounces, one must solve the coupled eqs. (2.15) and (2.16) with the boundary conditions described above.

\subsection{Sub-Planckian approximation to the bounce}

The problem can be simplified by using the low-energy approximation, which, as explained at the beginning of this section, is not physically restrictive if one works within the regime of validity of Einstein gravity (as we do). We illustrate now such an approximation.

The low-energy approximation consists in assuming that gravity is weak in the sense that

$$
H, \frac{1}{R} \ll M_{\mathrm{Pl}},
$$

where $R$ is the size of the bounce $(1 / R$ is roughly given by the mass scale that appears in the scalar potential $V$ ). The two conditions arise because gravitational corrections are

\footnotetext{
${ }^{3}$ The Coleman bounce is $\mathrm{O}(4)$-symmetric. The Hawking-Moss bounce, having a constant $h$, has the full $\mathrm{O}(5)$ symmetry of de Sitter space. Vacuum decay at finite-temperature $T$ is described by a configuration with period $1 / T$ in the Euclidean time coordinate. At large $T$, the thermal bounce becomes constant in time, acquiring a $\mathrm{O}(3) \otimes \mathrm{O}(2)$ symmetry (see [4] for a recent discussion). These are different solutions: a de Sitter space with Hubble constant $H$ is qualitatively similar but not fully equivalent to a thermal bath at temperature $T=H / 2 \pi$.
} 
suppressed by powers of the Planck mass, and are thereby small if the massive parameters of the problem are small in Planck units. During inflation, the condition on $H$ is satisfied in view of the experimental constraint $H<3.6 \times 10^{-5} \bar{M}_{\mathrm{Pl}}$ (see section 5.1 of [28]). The first condition is also not restrictive because it is necessary to avoid energies of order of the Planck scale, for which Einstein's theory breaks down.

Assuming that these conditions are satisfied, we expand $h$ and $\rho$ in powers of $\kappa=1 / \bar{M}_{\mathrm{Pl}}^{2}$ :

$$
h(r)=h_{0}(r)+\kappa h_{1}(r)+\mathcal{O}\left(\kappa^{2}\right), \quad \rho(r)=\rho_{0}(r)+\kappa \rho_{1}(r)+\mathcal{O}\left(\kappa^{2}\right) .
$$

The leading-order metric corresponds to de Sitter space:

$$
\rho_{0}(r)=\frac{\sin (H r)}{H} \text {. }
$$

Furthermore, we are interested in a situation in which $H \sim 1 / R$ : otherwise one can neglect $H$ and return to the flat space approximation discussed in $[4,18]$. Thus we are in a regime in which the vacuum energy is dominated by $V_{0}=3 H^{2} \bar{M}_{\mathrm{Pl}}^{2} \gg \delta V(h)$ and the gravitational background is perturbed only slightly by the bounce $h(r)$.

The equation of motion of the zeroth order bounce $h_{0}(r)$ (that is the equation on the de Sitter non-dynamical background) and the first correction $\rho_{1}$ to the metric function can be obtained by inserting the expansion of eq. (2.24) in eqs. (2.15) and (2.16). The de Sitter bounce $h_{0}(r)$ at zeroth order in $\kappa$ is given by

$$
h_{0}^{\prime \prime}+3 H \cot (H r) h_{0}^{\prime}=\frac{d V_{H}\left(h_{0}\right)}{d h},
$$

where $V_{H}$ is given in eq. (2.19), where $H$ can be evaluated at $h=0$ rather than at $h_{\max }$ : since the difference is an higher order effect in $\kappa$, we avoid introducing two different symbols. ${ }^{4}$ The boundary conditions are

$$
h^{\prime}(0)=h^{\prime}(\pi / H)=0 .
$$

At this lowest order, the effect of a general non-minimal coupling to gravity $f(h)$ is equivalent to replacing the potential $V(h)$ with the modified potential $V_{H}(h)$ given in eq. (2.19). The equation for $\rho_{1}$ is

$$
\left(\frac{\rho_{1}}{\cos (H r)}\right)^{\prime}=\frac{\tan ^{2} H r}{6 H^{2}}\left(\frac{h_{0}^{\prime 2}}{2}-\delta V\left(h_{0}\right)+3 H^{2} f\left(h_{0}\right)-\frac{3 H}{\tan H r} \frac{d f\left(h_{0}\right)}{d h} h_{0}^{\prime}\right)
$$

such that $\rho_{1}(r)$ can be obtained by solving either by integration starting from $\rho_{1}(0)=0$ (although some care is needed to handle apparent singularities at $r H=\pi / 2)$, or by converting

\footnotetext{
${ }^{4}$ As an aside comment, an $\mathrm{O}(4)$-symmetric space is conformally flat, such that by performing a Weyl transformation one can revert to flat-space equations with a Weyl-transformed action. A de Sitter space is conformally flat when written in terms of conformal time $\tilde{r} \equiv 2 \tan (H r / 2) / H$. Performing the associated Weyl transformation $h_{0}(r)=\tilde{h}_{0}(\tilde{r}) / \cos ^{2}(H r / 2)$, the bounce equation (2.26) becomes

$$
\frac{d^{2} \tilde{h}_{0}}{d \tilde{r}^{2}}+\frac{3}{\tilde{r}} \frac{d \tilde{h}_{0}}{d \tilde{r}}=\frac{\tilde{V}^{(1)}\left(\tilde{h}_{0}\left(1+H^{2} \tilde{r}^{2} / 4\right)\right)}{\left(1+H^{2} \tilde{r}^{2} / 4\right)^{3}}, \quad \tilde{V} \equiv V_{H}-H^{2} h^{2}=V-H^{2}\left(6 f+h^{2}\right)
$$

where $\tilde{V}^{(n)}$ is the $n$-th derivative of $\tilde{V}$.
} 
eq. (2.29) into a 1st-order linear differential equation that can be solved numerically. In the limit where the bounce has a size $R$ much smaller than $1 / H$, the solution

$$
\rho_{1}(r) \stackrel{R \ll 1 / H}{\simeq} \cos (H r) \int_{0}^{r} d r \frac{r^{2}}{6}\left(\frac{1}{2} h_{0}^{\prime 2}-\delta V\left(h_{0}\right)-\frac{3}{r} \frac{d f\left(h_{0}\right)}{d h} h_{0}^{\prime}\right)
$$

reduces to the flat-space solution of [4], times the overall $\cos (\mathrm{Hr})$ factor.

Our goal now is to compute the action (difference) $\mathcal{S}$ of eq. (2.10) because, which is the quantity that appears in the decay rate. The expansion for the fields in (2.24) leads to a corresponding expansion of $\mathcal{S}$ in powers of $\kappa$ :

$$
\mathcal{S}=\mathcal{S}_{0}+\kappa \mathcal{S}_{1}+\mathcal{O}\left(\kappa^{2}\right)
$$

The zeroth order action is ${ }^{5}$

$$
\mathcal{S}_{0}=2 \pi^{2} \int_{0}^{\pi / H} d r \frac{\sin ^{3} H r}{H^{3}}\left[\frac{h_{0}^{\prime 2}}{2}+\delta V_{H}\left(h_{0}\right)\right] .
$$

The leading correction due to gravity, $\Delta \mathcal{S}_{\text {gravity }} \equiv \kappa \mathcal{S}_{1}$, is

$$
\begin{aligned}
\Delta \mathcal{S}_{\text {gravity }}= & \frac{6 \pi^{2}}{\bar{M}_{\mathrm{Pl}}^{2}} \int_{0}^{\pi / H} d r\left[\frac{\sin ^{2}(H r)}{H^{2}} \rho_{1}\left(\frac{h_{0}^{\prime 2}}{2}+\delta V\left(h_{0}\right)-3 H^{2} f\left(h_{0}\right)\right)-\frac{\sin (H r)}{H} \rho_{1}^{\prime 2}+\right. \\
& \left.+2 H \sin (H r) \rho_{1}^{2}+\frac{\sin ^{2}(H r)}{H^{2}} f\left(h_{0}\right)\left(2 H \cot (H r) \rho_{1}^{\prime}+\rho_{1}^{\prime \prime}\right)\right]
\end{aligned}
$$

The expression of $\Delta \mathcal{S}_{\text {gravity }}$ above has been simplified by using the equation of $h_{0}$ in $(2.26)$ and by an integration by parts. It can be further simplified as follows. Rescaling $\rho_{1}(r) \rightarrow s \rho_{1}(r)$ corresponds to shifting $\rho_{1}(r)$ by $(s-1) \rho_{1}(r)$. By noticing that $(s-1) \rho_{1}(r)$ is a particular variation $\delta \rho_{1}$ we conclude that the action must have an extremum at $s=1$. Applying this argument to eq. (2.33) relates the integrals of terms linear and quadratic in $\rho_{1}$. The final simplified expression is:

$$
\Delta \mathcal{S}_{\text {gravity }}=\frac{6 \pi^{2}}{\bar{M}_{\mathrm{Pl}}^{2}} \int_{0}^{\pi / H} d r \frac{\sin (H r)}{H}\left[\rho_{1}^{\prime 2}-2 H^{2} \rho_{1}^{2}\right] .
$$

Note that the upper integration limit is simply $\pi / H$. In deriving it we have taken into account the dynamics of the spacetime volume: the shift in $r_{\max }$ does not affect the integral because the integrand contains a function, $\sin \mathrm{Hr}$, which vanishes at the integration boundaries. ${ }^{6}$

\footnotetext{
${ }^{5}$ The action contains the curvature term enhanced by negative powers of the Planck mass. The $\mathcal{O}(1 / \kappa)$ term cancels in the difference defining $\mathcal{S}$, eq. (2.10). Moreover, it leads to a term involving $\rho_{1}$ in the integrand of $\mathcal{S}_{0}$ proportional to $\left(\sin (H r)^{2} \rho_{1}^{\prime}\right)^{\prime}$. However, this total-derivative term gives no contribution to $\mathcal{S}_{0}$ for a $\rho_{1}^{\prime}$ that is regular at $r=0$ and $\pi / H$.

${ }^{6}$ The correction to $\rho$ generates a corresponding correction in $r_{\max }$, defined around eq. (2.17). Indeed

$$
0=\rho\left(r_{\max }\right)=\rho_{0}\left(r_{\max }\right)+\kappa \rho_{1}\left(r_{\max }\right)+\mathcal{O}\left(\kappa^{2}\right)
$$

tells us that $r_{\max }$ is a function of $\kappa$ that can be expanded around $\kappa=0: r_{\max }=\pi / H+\kappa r_{1}+\mathcal{O}\left(\kappa^{2}\right)$. By inserting the last expansion in eq. (2.35) we obtain $\kappa \rho_{0}^{\prime}(\pi / H) r_{1}+\kappa \rho_{1}(\pi / H)+\mathcal{O}\left(\kappa^{2}\right)=0$. Noticing that $\rho_{0}^{\prime}(\pi / H)=-1$, we find $r_{1}=\rho_{1}(\pi / H)$, where $\rho_{1}(\pi / H)$ can be obtained from the solution of eq. (2.29).
} 
In the limit $H \rightarrow 0$, eq. (2.34) reduces to the flat-space expression found in [4], which is positive-definite, unlike the result for generic $H$. Just like on flat space, $\Delta \mathcal{S}_{\text {gravity }}$ is independent of $h_{1}$ on-shell: the reason is that the only way $h_{1}$ could appear at first-order in $\kappa$ is by taking the first variation of the $h$-dependent part of the action, eq. (2.12), but this vanishes when $h_{0}$ solves eq. (2.26).

In conclusion, eqs. (2.26), (2.29), (2.32) and (2.34) tell us that, in order to compute the semiclassical decay rate including the first-order gravitational corrections, one just needs to compute the bounce $h_{0}$ on the background de Sitter space. This is easier than solving the coupled equations for the bounce and the geometry in eqs. (2.15) and (2.16). Being a onedimensional problem, it can be solved through an over-shooting/under-shooting method. Then, one needs to plug $h_{0}$ in the expression for $\rho_{1}$ to get $\mathcal{S}_{0}+\Delta \mathcal{S}_{\text {gravity. }}$. One can therefore focus on the equation of $h_{0}$. Imposing the boundary conditions in eq. (2.28) leads to well-defined solutions, as we will show in the next sections.

\section{Renormalizable potential}

In order to understand the influence of the de Sitter background on vacuum decay, we perform a numerical study of the problem for a toy renormalizable potential

$$
V(h)=V_{0}+\frac{M^{2}}{2} h^{2}-\frac{A}{3} h^{3}+\frac{\lambda}{4} h^{4}
$$

with $M^{2}=\lambda h_{\max } h_{\text {true }}$ and $A=\lambda\left(h_{\max }+h_{\text {true }}\right)$, such that the potential has a maximum at $h=h_{\max }$ and two vacua at $h=0$ and at $h=h_{\text {true: }}$ the latter vacuum is the true deeper vacuum provided that $h_{\text {true }}>2 h_{\max }>0$. Quantum corrections are perturbatively small when $\lambda \ll 4 \pi$ and $A \ll 4 \pi M$. The curvature of the potential at its maximum is $\mu^{2} \equiv-V^{(2)}\left(h_{\max }\right)=\lambda\left(h_{\text {true }}-h_{\max }\right) h_{\max }$. The constant term $V_{0}$ gives a Hubble constant $H$ through eq. (2.8).

\subsection{Zeroth order in $H, M \ll M_{\mathrm{Pl}}$}

The main qualitative influence of the Hubble rate $H$ on vacuum decay is most easily understood at zeroth order in the sub-Planckian expansion, ignoring the gravitational corrections that will be discussed in the next section.

We consider a typical illustrative example: vanishing non-minimal coupling to gravity (introduced later), $\lambda=0.6$ and $h_{\text {true }}=3 h_{\text {max }}$. In figure 1 we show the resulting bounces $h_{0}(t)$ at zeroth order in $H, M \ll M_{\mathrm{Pl}}$ for increasing values of $H$.

For $H \ll M$ the de Sitter radius $1 / H$ is much larger than the scale of the flat-space bounce, of order $1 / M$. Thereby, the flat space bounce is negligibly affected by the curvature of the space, fitting comfortably into a horizon.

We see that the critical value above which $H$ starts influencing the bounce action is of order $M$. Thereby the bounce correction to the energy density is of order $M^{4}$, which is negligible with respect to $V_{0}=3 \bar{M}_{\mathrm{Pl}}^{2} H^{2}$. This confirms that, in the relevant range, the bounce correction to the background is negligible, being Planck suppressed, so that it makes sense to first consider the zeroth-order approximation. 

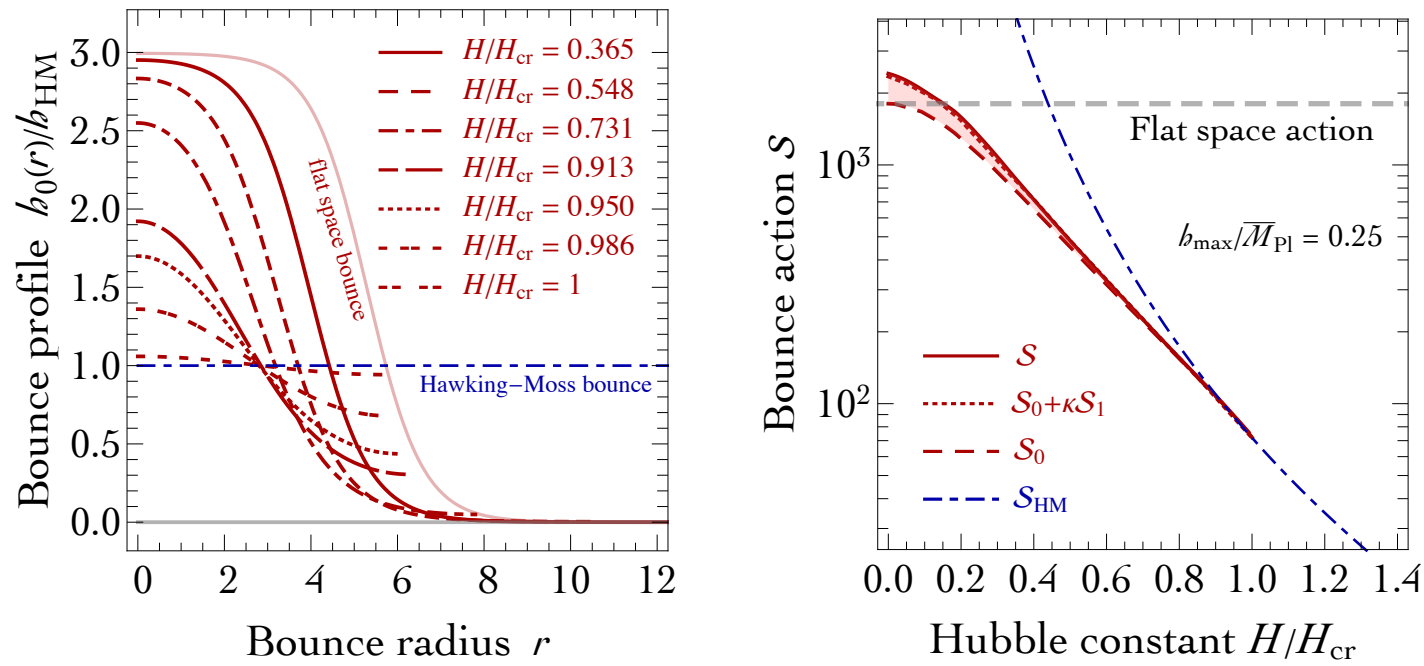

Figure 1. We consider the renormalizable quartic potential of eq. (3.1) for quartic scalar coupling $\lambda=0.6, h_{\text {true }}=3 h_{\max }$ and vanishing non-minimal coupling to gravity. The results exhibit the typical features of the general case. The left panel shows the bounce $h_{0}(r)$ for different values of the Hubble constant $H$. As $H$ is increased, the Coleman flat-space bounce gradually tends towards the Hawking-Moss bounce, until only the Hawking-Moss solution remains at $H>H_{\mathrm{cr}}$. The right panel shows the Coleman and Hawking-Moss actions, comparing our weak-gravity expansion with the full numerical result. Figure 2 shows the actions of the extra multi-bounce solutions.

Figure 1a shows that, increasing $H$, the flat-space bounce flattens and it tends to the constant Hawking-Moss bounce $h_{\mathrm{HM}}(r)=h_{\max }$ above a finite critical value $H_{\mathrm{cr}}$ of $H$, of order $M{ }^{7}$

The critical value of $H$ can be analytically computed by approximating the potential as a quadratic Taylor series in $h$ around its maximum: $V(h) \simeq V\left(h_{\max }\right)+\frac{1}{2} V^{(2)}\left(h_{\max }\right)\left(h-h_{\max }\right)^{2}$, such that the bounce equation at lowest order in $\kappa$ becomes

$$
h_{0}^{\prime \prime}(r)+3 H \cot (H r) h_{0}^{\prime}(r) \simeq V^{(2)}\left(h_{\max }\right)\left[h_{0}(r)-h_{\max }\right] .
$$

This linear equation is solved by

$$
h_{0}(r)-h_{\max } \propto \frac{P_{n}^{1}(\cos (H r))}{\sin (H r)} \quad \text { where } \quad n=\frac{1}{2}\left(\sqrt{9-\frac{4 V^{(2)}\left(h_{\max }\right)}{H^{2}}}-1\right)
$$

is the order of the Legendre function $P_{n}^{1}$; the other independent solution is not regular in $r=0$. The solution diverges at $\pi / H$ unless $n$ is integer. For $n=1$ one gets the constant Hawking-Moss solution. The first non constant solution, $h_{0}(r)-h_{\max } \propto \cos (H r)$, arises for $n=2$, corresponding to the critical value

$$
H_{\mathrm{cr}} \equiv \sqrt{-V^{(2)}\left(h_{\max }\right)} / 2 .
$$

\footnotetext{
${ }^{7}$ The thin-wall approximation approximates the bounce as two different constants, at $r<R$ and $r>R$. In cases where the thin-wall approximation holds in flat space at $H \ll M$, it ceases to be valid as $H$ is increased. Thereby the continuous transition from the Coleman bounce to the Hawking-Moss bounce is not visible in the thin-wall approximation [23, 29]. We emphasize that the Hawking-Moss bounce is not an approximation to the Coleman bounce: they are two different solutions.
} 


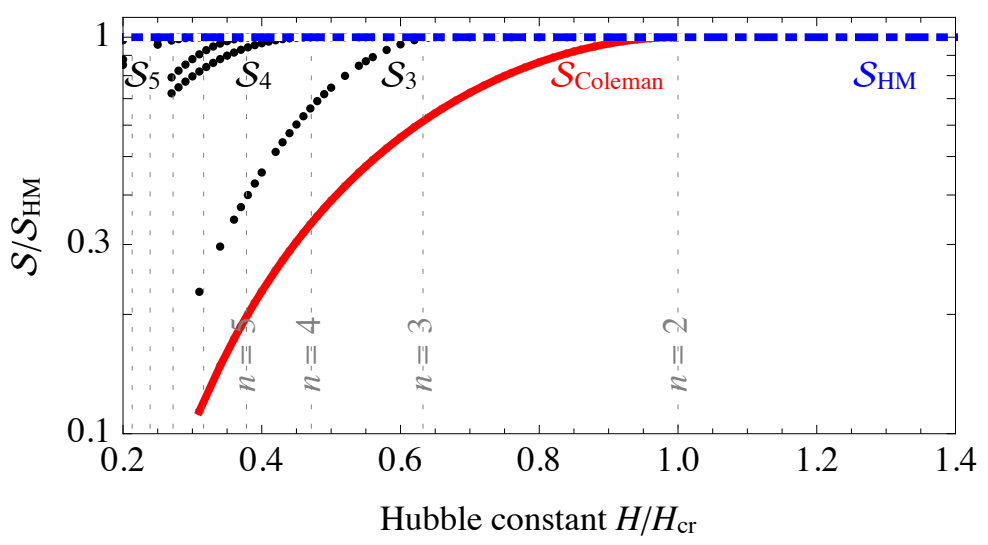

Figure 2. For the renormalizable quartic potential considered in figure 1, we show the actions of the multi-bounce solutions in units of the Hawking-Moss action.

For values of $H$ close to $H_{\mathrm{cr}}$, by expanding the potential to higher orders around its maximum, one finds that Coleman bounces must satisfy

$$
\Delta \equiv 4+\frac{V^{(2)}\left(h_{\max }\right)}{H^{2}}=-\frac{\left(h_{0}(0)-h_{\max }\right)^{2}}{14 H^{2}}\left[V^{(4)}\left(h_{\max }\right)+\frac{V^{(3)}\left(h_{\max }\right)^{2}}{12 H^{2}}\right]
$$

and their action is $\mathcal{S}_{\text {Coleman }} \simeq \mathcal{S}_{\mathrm{HM}}+2 \pi^{2}\left(h_{0}(0)-h_{\max }\right)^{2} \Delta / 15 H^{2}$ [30]. The sign of $\Delta$ is fixed by the potential, so that Coleman bounces exist for $H<H_{\text {cr }}$ when $\Delta<0$, and for $H>H_{\text {cr }}$ otherwise. $^{8}$ Our potential has $V^{(4)}>0$ and thereby $\Delta<0$, so that Coleman bounces exist only for $H<H_{\mathrm{cr}}$.

Higher values of $n \geq 3$ correspond to bounces that cross the top of the potential $n-1$ times and exist for $H \leq \sqrt{-V^{(2)}\left(h_{\max }\right) /\left(n^{2}+n-2\right)}$. They never dominate the path-integral, as their action is between the Coleman action and the Hawking-Moss action, as shown in figure 2. For $H \ll \sqrt{-V^{(2)}\left(h_{\max }\right)}$ their actions, $\mathcal{S}_{n}$, are multiple integers of the Coleman action, allowing the resummation of their contributions in the dilute-gas approximation [26, 27]. As $H$ grows, multi-bounce solutions progressively no longer fit into the Euclidean de Sitter space.

\subsection{First order in $H, M \ll M_{\mathrm{Pl}}$}

figure $1 \mathrm{~b}$ shows the bounce actions. In order to compute them, including gravitational corrections, we need to fix the overall mass scale of the potential. We choose a mildly sub-Planckian value of the field value of the top of the potential, $h_{\max }=0.25 \bar{M}_{\mathrm{Pl}}$, in order to have mild Planck-suppressed corrections.

The Hawking-Moss bounce exists whenever the potential has a barrier, and its action can be easily computed exactly. When the Hubble constant is much smaller than the

\footnotetext{
${ }^{8}$ The expansion of the potential fails for different potentials that involve vastly different mass scales (in particular the ones with a very flat barrier), which need a more careful analysis of higher order terms in eq. (3.2). In particular, if $V^{(2)}\left(h_{\max }\right)$ vanishes, it gets replaced by an average around the top of the barrier [30-32].
} 


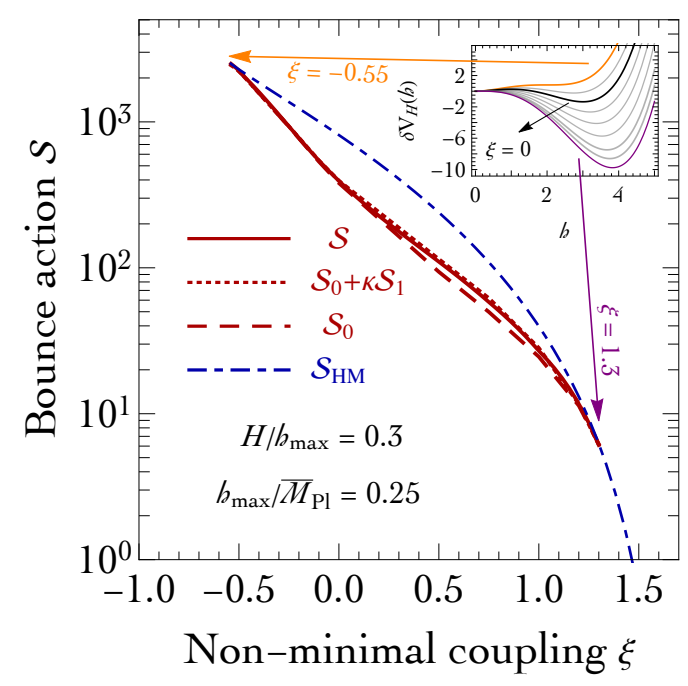

Figure 3. Coleman and Hawking-Moss bounce actions computed after adding a non-minimal coupling to gravity $f=\xi h^{2}$ to the model considered in figure 1 . Its main effect is to modify the effective potential, so that the true vacuum disappears for negative large $\xi$, and the potential barrier disappears for positive large $\xi$. Gravitational corrections to the action are again well approximated by our sub-Planckian expansion.

inverse size of the bounce, the Hawking-Moss bounce becomes negligible because its action (plotted in blue) becomes large.

The Coleman bounce only exists for sub-critical values of $H$, and its action (plotted in red) smoothly merges with the Hawking-Moss action for $H=H_{\mathrm{cr}}$. We plotted 3 different results for its action:

1. The continuous curve is the full numerical result $\mathcal{S}$.

2. The dashed curve is $\mathcal{S}_{0}$, the action at zeroth order in $\kappa=1 / \bar{M}_{\mathrm{Pl}}^{2}$. We see that it already provides a reasonably accurate approximation.

3. The dotted curve is the first-order approximation $\mathcal{S}_{0}+\kappa \mathcal{S}_{1}$, and it almost coincides with the exact numerical curve.

\subsection{Effect of non-minimal couplings}

Finally, the effect of a non-minimal coupling to gravity $f(h)$ can be trivially discussed at zeroth order in the sub-Planckian limit, being just equivalent to a shift in the scalar potential $V \rightarrow V_{H}=V-6 H^{2} f$, see eq. (2.26). This effect is not present in flat space, where the Hubble constant vanishes, $H=0$.

For example a non-minimal coupling to gravity $f(h)=\xi h^{2}$, governed by a dimensionless coupling $\xi$, is equivalent to a shift $M^{2} \rightarrow M_{H}^{2} \equiv M^{2}-12 \xi H^{2}$ in our renormalizable quartic potential of eq. (3.1). This is just a redefinition of the potential parameters which makes the qualitative implications obvious, in agreement with the full numerical result shown in figure 3 : 
- A positive $\xi>0$ reduces $M_{H}^{2}<M^{2}$ and thereby the potential barrier, decreasing the actions and increasing the tunnelling rate (see also [23]). The critical value $H_{\text {cr }}$ depends on $\xi$, so that by increasing $\xi$ one first violates the condition $H<H_{\mathrm{cr}}$, leading to the disappearance of Coleman bounces. A larger $\xi$ leads to $M_{H}^{2}<0$, so that the potential barrier disappears and the false vacuum is classically destabilised: this corresponds to $\mathcal{S}_{\mathrm{HM}}=0$ in figure 3 .

- A negative $\xi$ has the effect of increasing the potential barrier. Ultimately, a too large negative $\xi$ destabilizes the true vacuum: at this point $V\left(h_{\text {true }}\right)=V\left(h_{\max }\right)$, such that the Coleman bounce becomes equal to the Hawking-Moss bounce.

In figure 3 we also depict the full numerical action assuming a mildly sub-Planckian potential with $h_{\max }=0.25 \bar{M}_{\mathrm{Pl}}$ : our sub-Planckian expansion again reproduces the full numerical result. The above simplicity is lost if Planckian energies are involved; however in such a case Einstein gravity cannot be trusted.

\section{Standard Model vacuum decay during inflation}

Finally, we apply our general formalism to the case of physical interest: instability of the electro-weak SM vacuum during inflation.

The probability per unit time and volume of vacuum decay during inflation can be estimated on dimensional grounds as $d \wp / d V d t \sim H^{4} \exp (-\mathcal{S})$, where $\mathcal{S}$ is the action of the relevant bounce configuration. The total probability of vacuum decay during inflation then is $\wp \sim T L^{3} H^{4} \exp (-\mathcal{S})$, corresponding to a total time $T \sim N / H$ and volume $L^{3} \sim H^{-3} \exp (3 N)$, such that $\wp \sim \exp (3 N-\mathcal{S})$. Therefore, a small 'probability' $\wp \sim 1$ of vacuum tunnelling during inflation needs a bounce action $\mathcal{S} \gtrsim 3 N .^{9}$ The horizon of the visible universe corresponds to a minimal number of $N \sim 60 e$-foldings of inflation.

The computation of the bounce actions $\mathcal{S}$ in the SM case needs to take into account the peculiar features of the SM Higgs potential, which is nearly scale-invariant and can be approximated as

$$
V(h) \approx \lambda(h) \frac{h^{4}}{4} \approx-b \ln \left(\frac{h^{2}}{h_{\mathrm{cr}}^{2} \sqrt{e}}\right) \frac{h^{4}}{4},
$$

where the running Higgs quartic can turn negative, $\lambda(h)<0$, at large field values. This happens for the present best-fit values of $M_{t}, M_{h}$ and $\alpha_{3}$, that lead to $h_{\mathrm{cr}}=5 \times 10^{10} \mathrm{GeV}[18$, 33, 34]. The $\beta$-function of $\lambda$ around $h_{\mathrm{cr}}$ can be approximated as $b \approx 0.15 /(4 \pi)^{2}$.

Furthermore, we add a nonminimal coupling of the Higgs field to gravity $f(h)=\xi_{H} h^{2}$, such that the effective potential of eq. (2.19) relevant in the sub-Planckian limit is

$$
V_{H}=V-12 \xi_{H} H^{2} \frac{h^{2}}{2} .
$$

Finally, we assume that inflation can be approximated as an extra constant term $V_{0}$ in the potential. A possible extra quartic scalar coupling of the inflaton to the Higgs would

\footnotetext{
${ }^{9}$ Evading this bound trough anthropic selection would need a very special landscape of unstable vacua with no low-scale inflation.
} 
manifest itself as an extra contribution to the effective Higgs mass term in eq. (4.2), which is equivalent to a modified effective $\xi_{H}$.

\subsection{SM vacuum decay for small $H$}

In the limit of small $H$ one can view curvature, gravity and quantum effects as perturbative corrections to the simple approximation of a constant $\lambda<0$. Perturbing around a potential with no barrier and no true vacuum requires a careful understanding [35]. Kinetic energy acts as a barrier, such that the dimension-less potential admits a continuous family of flat-space bounces, parameterized by their arbitrary scale $R$ :

$$
h_{0}(r)=\sqrt{\frac{2}{|\lambda|}} \frac{2 R}{r^{2}+R^{2}} \quad(H=0) .
$$

The action of these 'Fubini' bounces is $\mathcal{S}=8 \pi^{2} / 3|\lambda|$ [3]. Minimal gravitational couplings have been included numerically in [36, 37]; in this section we will apply our analytic subPlanckian approximation to take into account gravity (including non-minimal couplings).

Quantum corrections can be included roughly by renormalizing the quartic coupling at the scale of the bounce, $\mathcal{S} \approx 8 \pi^{2} / 3|\lambda(1 / R)|$ [35]. For the best-fit values of the SM parameters, the Higgs quartic $\lambda$ runs in such a way that tunnelling is dominated by mildly subPlanckian bounces, such that Planck-suppressed corrections are small in flat space [3, 4].

We now include curvature and gravitational effects, assuming $H \ll 1 / R$. Performing the integral in eq. (2.30) we obtain the leading correction in small $H R$ to the metric:

$$
\rho_{1}(r)=\cos (H r) \frac{1+6 \xi_{H}}{3|\lambda| R}\left(\frac{r R\left(r^{2}-R^{2}\right)}{\left(r^{2}+R^{2}\right)^{2}}+\arctan \left(\frac{r}{R}\right)\right) .
$$

The gravitational corrections to the action combine with the quantum corrections (see also [17]) in order to give the final formula valid for $H \ll 1 / R \ll M_{\mathrm{Pl}}$ :

$$
\mathcal{S} \simeq \min _{R \ll 1 / H}\left[\frac{8 \pi^{2}}{3|\lambda(1 / R)|}\left(1+6\left(1+6 \xi_{H}\right)(H R)^{2} \ln H R\right)+\frac{32 \pi^{2}\left(1+6 \xi_{H}\right)^{2}}{45\left(R \bar{M}_{\mathrm{Pl}} \lambda(1 / R)\right)^{2}}\right] .
$$

This expression only holds when the corrections are small. In this regime, the vacuum decay rate during inflation is negligible, having a rate similar to the rate in the longer post-inflationary phase.

\subsection{SM vacuum decay for large $H \gg h_{\mathrm{cr}}$}

The interesting case that can lead to possibly significant inflationary enhancements of the tunnelling rate corresponds to values of $H$ comparable or larger than the inverse size $1 / R$ of the bounce, so that the approximation used in the previous section 4.1 does not apply.

For simplicity, we start by discussing the opposite limit, in which the Hubble constant $H$ during inflation is sub-Planckian and much larger than the critical scale $h_{\text {cr }}$ above which the Higgs quartic coupling turns negative. Then, we can approximate the SM potential at field values around $h \sim H$ as a quartic potential, with a constant negative $\lambda$ renormalized 
around $H$. Adding the non-minimal coupling to gravity $f(h)=\xi_{H} h^{2}$ gives the effective potential relevant in the sub-Planckian limit:

$$
\delta V_{H}=-12 \xi_{H} H^{2} \frac{h^{2}}{2}+\lambda \frac{h^{4}}{4} .
$$

Scale invariance is broken by the de Sitter background with Hubble constant $H$, so that the bounce action can only depend on the dimensionless parameters $\xi_{H}$ and $\lambda$. By performing the field redefinition $h(r) \rightarrow \alpha h(r)$, where $\alpha$ is a constant, one obtains $\mathcal{S}\left(\xi_{H}, \lambda\right)=\alpha^{2} \mathcal{S}\left(\xi_{H}, \alpha^{2} \lambda\right)$ which implies $\mathcal{S} \propto 1 / \lambda$. Therefore, we only need to compute $\mathcal{S}$ as function of $\xi_{H}$.

A potential barrier exists for $\xi_{H}<0$ : then $h_{\max }=H \sqrt{12 \xi_{H} / \lambda}$ and Hawking-Moss bounces have action $\mathcal{S}_{\mathrm{HM}}=-96 \pi^{2} \xi_{H}^{2} / \lambda$.

According to the argument in section 3.1, the critical value of $H$ that controls the existence of Coleman bounces is $H_{\mathrm{cr}}=\sqrt{-V^{(2)}\left(h_{\max }\right)} / 2$. For the potential of eq. (4.6) this means $H / H_{\text {cr }}=1 / \sqrt{-6 \xi_{H}}$, so that $H=H_{\text {cr }}$ for $\xi_{H}=-1 / 6$. As discussed in section 3.1, Coleman bounces exist for $H<H_{\text {cr }}$ or $H>H_{\text {cr }}$ depending on the sign of the higherorder coefficient $\Delta$ defined in eq. (3.5). In the present case $\Delta \propto-\lambda\left(1+6 \xi_{H}\right)$, with a positive proportionality constant. This potential behaves in an unusual way: $\Delta$ vanishes at the critical value $\xi_{H}=-1 / 6$, for which Hawking-Moss bounces have the same action $\mathcal{S}=-8 \pi^{2} / 3 \lambda$ as flat-space Fubini bounces. Flat-space bounces are relevant because the action is Weyl invariant for $\xi_{H}=-1 / 6$, such that de Sitter space is conformally equivalent to flat space. Indeed, the Weyl-transformed eq. (2.27) reduces to $\tilde{h}_{0}^{\prime \prime}+3 \tilde{h}_{0}^{\prime} / \tilde{r}=\lambda \tilde{h}_{0}^{3}$, satisfied by Fubini bounces $\tilde{h}_{0}=\sqrt{2 /|\lambda|} 2 R /\left(R^{2}+\tilde{r}^{2}\right)$, where $R$ is an arbitrary constant. By Weylrescaling them back to the original field $h_{0}$ and coordinate $r$ we obtain the Coleman bounce for $\xi_{H}=-1 / 6$, and thereby $H=H_{\mathrm{cr}}$ :

$$
h_{0}(r)=\frac{\sqrt{2 /|\lambda|} H^{2} R}{1+H^{2} R^{2} / 4-\left(1-H^{2} R^{2} / 4\right) \cos H r} .
$$

For $R=2 / H$ this corresponds to constant Hawking-Moss bounces; for $R \ll 2 / H$ to Coleman bounces, for $R \gg 2 / H$ to Coleman bounces centred around $r=\pi / H$. For this special potential the convergence of Coleman bounces with Hawking-Moss bounces happens at $H=H_{\text {cr }}$ rather than gradually for $H \rightarrow H_{\text {cr }}$.

Eq. (4.7) together with de Sitter space actually solve the full gravitational equations (not only those in the sub-Planckian limit), as can be explicitly verified or understood from the argument given in flat space in footnote 6 of [4].

Figure 4 illustrates the situation. Extra multi-bounce solutions that perform multiple oscillations around the maximum with action $\mathcal{S} \geq \mathcal{S}_{\mathrm{HM}}$ appear below the extra critical values $\xi_{H}=\left(2-n-n^{2}\right) / 24$ with $n=\{3,4, \ldots\}$.

In conclusion, vacuum decay is safely slow if $\mathcal{S}_{\mathrm{HM}}>3 N$ which implies the condition

$$
-\xi_{H}<\sqrt{-\frac{N \lambda(H)}{32 \pi^{2}}} \approx-0.04 \sqrt{-\frac{\lambda(H)}{0.01}} \quad \text { for } H \gg h_{\mathrm{cr}}
$$




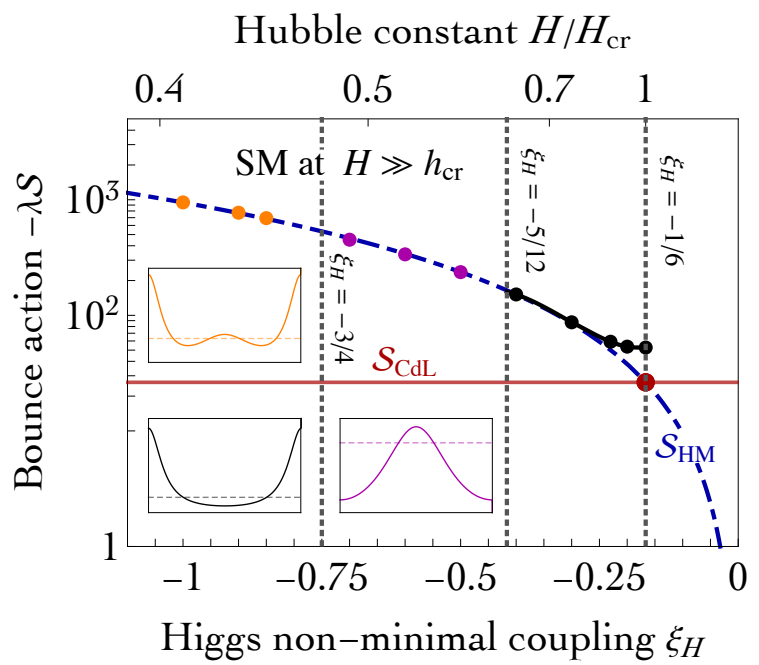

Figure 4. Bounce actions for the potential $V=\lambda h^{4} / 4$ with $\lambda<0$ and a non-minimal $\xi_{H}$ coupling to gravity. As the action is proportional to $1 / \lambda$, we plot the product $-\lambda \mathcal{S}$ as function of $\xi_{H}$. The red dot denotes the Coleman bounces of eq. (4.7). The other dots denote multi-bounces, as plotted in the insets.

\subsection{SM vacuum decay for $H \sim h_{\mathrm{cr}}$}

We finally consider the more complicated intermediate case where the Hubble constant $H$ during inflation is comparable to the instability scale $h_{\mathrm{cr}}$ of the SM potential. For best-fit values of the SM parameters, this scale is sub-Planckian, so that we can compute at zeroth order in $1 / \bar{M}_{\mathrm{Pl}}$ by solving the approximated bounce equation, eq. (2.26).

SM vacuum decay for $\boldsymbol{\xi}_{\boldsymbol{H}}=\mathbf{0}$. Let us first consider the case of vanishing non-minimal coupling of the Higgs to gravity. The approximated SM potential of eq. (4.1) has a maximum at $h_{\max }=h_{\mathrm{cr}}$. The action of the Hawking-Moss bounce is $\mathcal{S}_{\mathrm{HM}}=b \pi^{2} h_{\max }^{4} / 3 H^{4}$ and the critical value of $H$ is

$$
H_{\mathrm{cr}} \equiv \frac{\sqrt{-V^{(2)}\left(h_{\max }\right)}}{2}=\sqrt{\frac{b}{2}} h_{\max }=0.025 h_{\max } .
$$

At the critical value $H=H_{\text {cr }}$ where Coleman and Hawking-Moss solutions merge, vacuum decay is suppressed by large actions $\mathcal{S}_{\mathrm{HM}}=4 \pi^{2} / 3 b \approx 13000$, and the coefficient $\Delta$ that determines the behaviour of Coleman solutions is positive, such that Coleman bounces exist for $H>H_{\text {cr }}$ and are irrelevant in view of $\mathcal{S}_{\text {Coleman }}>\mathcal{S}_{\mathrm{HM}}$. Thereby, the bound on vacuum decay is dominated by Hawking-Moss bounces at $H>H_{\text {cr }}$. Imposing $\mathcal{S}_{\mathrm{HM}} \lesssim 3 N$ implies

$$
\frac{H}{h_{\max }} \lesssim\left(\frac{8 \pi^{2}}{9 N} \frac{V\left(h_{\max }\right)}{h_{\max }^{4}}\right)^{1 / 4}=\left(\frac{b \pi^{2}}{9 N}\right)^{1 / 4} \lesssim 0.06 .
$$

This bound can be compared with the bound $H / h_{\max } \lesssim 0.045$ derived in [18] by solving the Fokker-Planck or Langevin equation that describes the evolution in real time of the inflationary quantum fluctuations of the Higgs field. There is numerical agreement, even though the parametric dependence in eq. (4.10) does not match the one in [18]. Indeed [18] found 


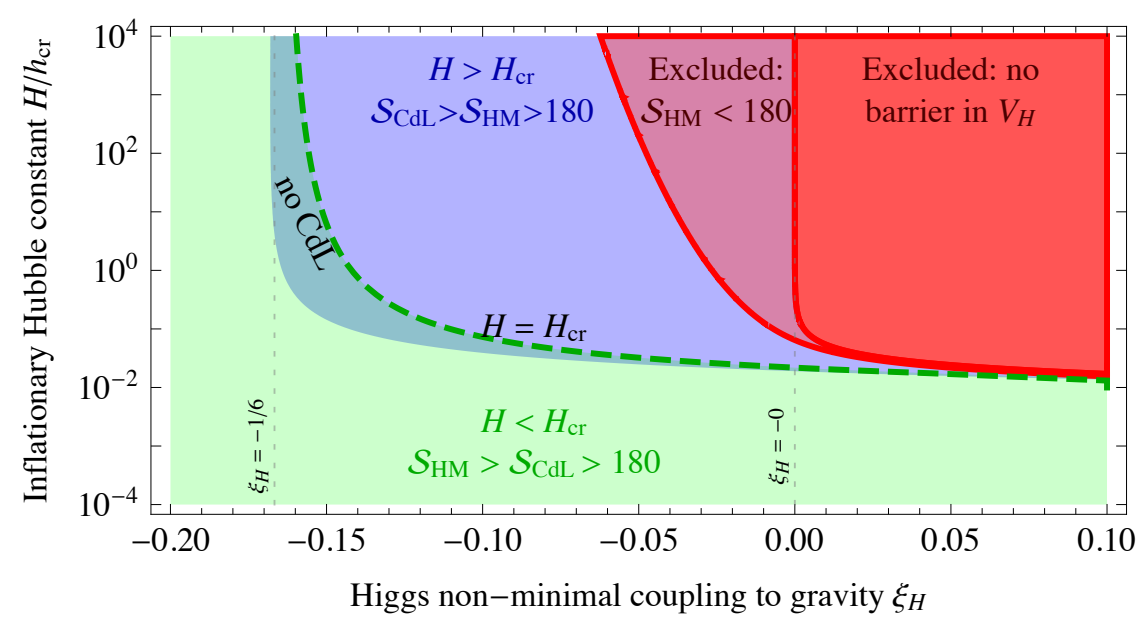

Figure 5. As a function of $\xi_{H}$ and of the Hubble constant in units of the instability scale $h_{\text {cr }}$ (and for $N=60 e$-folds of inflation), we plot: the allowed region where $H<H_{\text {cr }}$ in green with dashed boundary; the region where tunnelling is dominated by Hawking-Moss in blue; in red the excluded region where tunnelling is too fast. For $\xi_{H}<0$ this regions agrees with [18], while for $\xi_{H}>0$ our bounds are weaker.

that the Higgs acquires a Gaussian distribution with variance that grows with the number $N$ of $e$-foldings as $\sqrt{\left\langle h^{2}\right\rangle}=H \sqrt{N} / 2 \pi$, without approaching a limiting distribution. Thereby the dedicated study of [18] was necessary and cannot be reproduced by the Hawking-Moss tunnelling computations, which anyhow give a correct result, up to factors of order $\sqrt{N}$.

SM vacuum decay for $\xi_{H} \neq 0$. A non-minimal coupling of the Higgs to gravity, $\xi_{H} \neq 0$, is unavoidably generated by SM RGE running, and gives an extra contribution $M_{h}$ to the effective Higgs mass parameter in the effective inflationary potential $V_{H}$ of eq. (4.2), such that its maximum gets shifted from $h_{\max }=h_{\mathrm{cr}}$ to

$$
h_{\max }=H\left[-\frac{b}{12 \xi_{H}} W\left(-\frac{12 \xi_{H} H^{2}}{b h_{\mathrm{cr}}^{2}}\right)\right]^{-1 / 2}
$$

where $W(z)$ is the product-log function defined by $z=W e^{W}$ : it is real for $z>-1 / e$. Otherwise, the potential $V_{H}$ has no potential barrier because $M_{h}^{2}$ is too negative:

$$
M_{h}^{2}=-12 \xi_{H} H^{2}<-\frac{b h_{\max }^{2}}{e} \quad\left(\text { no barrier in } V_{H}\right) .
$$

The action of the Hawking-Moss bounce is

$$
\mathcal{S}_{\mathrm{HM}} \simeq \frac{8 \pi^{2}}{3} \frac{\delta V_{H}\left(h_{\max }\right)}{H^{4}}=\frac{48 \pi^{2} \xi_{H}^{2}}{b} \frac{1+2 W}{W^{2}} .
$$

The conditions that determine the relative role of Coleman and Hawking-Moss bounces around the critical situation $H=H_{\mathrm{cr}}$ give rise to a non-trivial pattern, plotted in figure 5 . The conclusion is again that only relevant vacuum decay bound is $\mathcal{S}_{\mathrm{HM}}>3 N$. In the limit $H \gg h_{\mathrm{cr}}$ this reduces to eq. (4.8). For generic values of $H$ the bound is plotted numerically 
in figure 5, where the red region is excluded because inflationary vacuum decay is too fast. For $\xi_{H}<0$ such bound agrees with the corresponding result of [18]. Indeed [18] found that a positive $M_{h}^{2}>0$ limits the Higgs fluctuations which, after a few $e$-foldings, converge towards a limiting distribution, well described by the Hawking-Moss transition. On the other hand, for $M_{h}^{2} \leq 0\left(\xi_{H} \geq 0\right)$ the Higgs fluctuations grow with $N$ (as $\sqrt{N}$ for $M_{h}^{2}=0$, and exponentially for $M_{h}^{2}<0$ ), so that the detailed dynamical study of [18] is needed. Nevertheless, for $\xi_{H}>0$, the bound $\mathcal{S}_{\mathrm{HM}}>3 N$, is almost numerically equivalent to the simpler bound on $M_{h}^{2}$ in eq. (4.12) that guarantees that $V_{H}$ has a potential barrier, which reads

$$
\frac{H}{h_{\max }} \lesssim\left(\frac{b}{12 e \xi_{H}}\right)^{1 / 2} \lesssim \frac{0.005}{\sqrt{\xi_{H}}} .
$$

figure 5 shows that this bound is weaker than the bound of [18]. A Langevin simulation performed along the lines of [18] agrees with our bound, while [18] made a simplifying approximation ('neglecting the small Higgs quartic coupling') which is not accurate around the bound at $\xi_{H}>0$.

\section{Conclusions}

In section 2 we developed a simplified formalism for computing vacuum decay from a de Sitter space with Hubble constant $H$, assuming that both $H$ and the mass scale of the scalar potential are sub-Planckian. This is not a limitation, given that otherwise Einstein gravity cannot anyhow be trusted. In this approximation, the bounce action is obtained as a power series in $1 / M_{\mathrm{Pl}}$, and a non-minimal scalar coupling to gravity can be reabsorbed in an effective scalar potential, see eq. (2.19).

In section 3 we considered a renormalizable single-field potential. We verified that our expansion reproduces full numerical result. Furthermore we found that, increasing $H$, the flat-space Coleman bounce continuously deforms into the Hawking-Moss bounce. Only the Hawking-Moss bounce exists above a critical value of the Hubble constant, equal to $H_{\mathrm{cr}}^{2}=-V^{(2)}\left(h_{\max }\right) / 4$. For $H<H_{\mathrm{cr}}$ the Coleman bounce appears and dominates vacuum decay, having a smaller action than the Hawking-Moss bounce. For smaller values of $H$ extra bounces that oscillate around the top of the barrier appear, but they never dominate the path-integral. In the flat space limit $H \rightarrow 0$ they reduce to the infinite series of multi-bounce solutions.

In section 4 we studied quantum tunnelling of the electroweak vacuum during inflation, assuming that the SM Higgs potential is unstable at large field values, as happens for present central values of the SM parameters. Coleman bounces are still connected to Hawking-Moss bounces, altough the fact that the SM potential has a negative quartic at large field values and is quasi-scale-invariant makes their relation different. We exhibit a limit where they are conformally equivalent. Anyhow we found that only HawkingMoss bounces imply a significant bound on vacuum decay during inflation. If the minimal coupling of the Higgs to gravity is negative, $\xi_{H}<0$, our tunnelling computation confirms previous upper bounds on $H$ obtained from statistical simulations (needed to address other cosmological issues). If $\xi_{H}>0$ we find weaker bounds, and explain why the approximation made in earlier works [18] is not accurate. 


\section{Acknowledgments}

We thank G. D'Amico, J.R. Espinosa, A. Rajantie, M. Schwartz and S.M. Sibiryakov for useful discussions. This work was supported by the ERC grant NEO-NAT. The work of A. Katsis is co-financed by the European Union (European Social Fund - ESF) and Greek national funds through the action "Strengthening Human Resources Research Potential via Doctorate Research" of State Scholarships Foundation (IKY), in the framework of the Operational Programme "Human Resources Development Program, Education and Lifelong Learning" of the National Strategic Reference Framework (NSRF) 2014-2020.

Open Access. This article is distributed under the terms of the Creative Commons Attribution License (CC-BY 4.0), which permits any use, distribution and reproduction in any medium, provided the original author(s) and source are credited.

\section{References}

[1] S.R. Coleman, The Fate of the False Vacuum. 1. Semiclassical Theory Phys. Rev. D 15 (1977) 2929 [Erratum ibid. D 16 (1977) 1248] [INSPIRE].

[2] S.R. Coleman and F. De Luccia, Gravitational Effects on and of Vacuum Decay, Phys. Rev. D 21 (1980) 3305 [inSPIRE].

[3] G. Isidori, V.S. Rychkov, A. Strumia and N. Tetradis, Gravitational corrections to standard model vacuum decay, Phys. Rev. D 77 (2008) 025034 [arXiv:0712.0242] [InSPIRE].

[4] A. Salvio, A. Strumia, N. Tetradis and A. Urbano, On gravitational and thermal corrections to vacuum decay, JHEP 09 (2016) 054 [arXiv: 1608.02555] [INSPIRE].

[5] G.W. Gibbons and S.W. Hawking, Cosmological Event Horizons, Thermodynamics, and Particle Creation, Phys. Rev. D 15 (1977) 2738 [InSPIRE].

[6] S.W. Hawking and I.G. Moss, Supercooled Phase Transitions in the Very Early Universe, Phys. Lett. B 110 (1982) 35 [INSPIRE].

[7] J.R. Espinosa, G.F. Giudice and A. Riotto, Cosmological implications of the Higgs mass measurement, JCAP 05 (2008) 002 [arXiv:0710.2484] [INSPIRE].

[8] A. Kobakhidze and A. Spencer-Smith, Electroweak Vacuum (In)Stability in an Inflationary Universe, Phys. Lett. B 722 (2013) 130 [arXiv:1301.2846] [INSPIRE].

[9] A. Kobakhidze and A. Spencer-Smith, The Higgs vacuum is unstable, arXiv:1404.4709 [INSPIRE].

[10] M. Fairbairn and R. Hogan, Electroweak Vacuum Stability in light of BICEP2, Phys. Rev. Lett. 112 (2014) 201801 [arXiv:1403.6786] [INSPIRE].

[11] K. Enqvist, T. Meriniemi and S. Nurmi, Higgs Dynamics during Inflation, JCAP 07 (2014) 025 [arXiv: 1404.3699] [INSPIRE].

[12] K. Kamada, Inflationary cosmology and the standard model Higgs with a small Hubble induced mass, Phys. Lett. B 742 (2015) 126 [arXiv:1409.5078] [INSPIRE].

[13] A. Hook, J. Kearney, B. Shakya and K.M. Zurek, Probable or Improbable Universe? Correlating Electroweak Vacuum Instability with the Scale of Inflation, JHEP 01 (2015) 061 [arXiv: 1404.5953] [INSPIRE]. 
[14] M. Herranen, T. Markkanen, S. Nurmi and A. Rajantie, Spacetime curvature and the Higgs stability during inflation, Phys. Rev. Lett. 113 (2014) 211102 [arXiv:1407.3141] [InSPIRE].

[15] J. Kearney, H. Yoo and K.M. Zurek, Is a Higgs Vacuum Instability Fatal for High-Scale Inflation?, Phys. Rev. D 91 (2015) 123537 [arXiv:1503.05193] [INSPIRE].

[16] W.E. East, J. Kearney, B. Shakya, H. Yoo and K.M. Zurek, Spacetime Dynamics of a Higgs Vacuum Instability During Inflation, Phys. Rev. D 95 (2017) 023526 [arXiv:1607.00381] [INSPIRE].

[17] A. Shkerin and S. Sibiryakov, On stability of electroweak vacuum during inflation, Phys. Lett. B 746 (2015) 257 [arXiv: 1503.02586] [INSPIRE].

[18] J.R. Espinosa et al., The cosmological Higgstory of the vacuum instability, JHEP 09 (2015) 174 [arXiv: 1505.04825] [INSPIRE].

[19] K. Kohri and H. Matsui, Higgs vacuum metastability in primordial inflation, preheating and reheating, Phys. Rev. D 94 (2016) 103509 [arXiv:1602.02100] [INSPIRE].

[20] A. Rajantie and S. Stopyra, Standard Model vacuum decay with gravity, Phys. Rev. D 95 (2017) 025008 [arXiv: 1606. 00849] [INSPIRE].

[21] K. Enqvist, M. Karciauskas, O. Lebedev, S. Rusak and M. Zatta, Postinflationary vacuum instability and Higgs-inflaton couplings, JCAP 11 (2016) 025 [arXiv: 1608. 08848] [INSPIRE].

[22] Y. Ema, M. Karciauskas, O. Lebedev and M. Zatta, Early Universe Higgs dynamics in the presence of the Higgs-inflaton and non-minimal Higgs-gravity couplings, JCAP 06 (2017) 054 [arXiv: 1703.04681] [INSPIRE].

[23] O. Czerwińska, Z. Lalak, M. Lewicki and P. Olszewski, The impact of non-minimally coupled gravity on vacuum stability, JHEP 10 (2016) 004 [arXiv: 1606.07808] [INSPIRE].

[24] S.K. Blau, E.I. Guendelman and A.H. Guth, The Dynamics of False Vacuum Bubbles, Phys. Rev. D 35 (1987) 1747.

[25] U. Nucamendi and M. Salgado, Scalar hairy black holes and solitons in asymptotically flat space-times, Phys. Rev. D 68 (2003) 044026 [gr-qc/0301062] [INSPIRE].

[26] J. Callan and S.R. Coleman, The Fate of the False Vacuum. 2. First Quantum Corrections, Phys. Rev. D 16 (1977) 1762 [INSPIRE].

[27] A. Andreassen, D. Farhi, W. Frost and M.D. Schwartz, Precision decay rate calculations in quantum field theory, Phys. Rev. D 95 (2017) 085011 [arXiv: 1604.06090] [INSPIRE].

[28] Planck collaboration, P.A.R. Ade et al., Planck 2015 results. XX. Constraints on inflation, Astron. Astrophys. 594 (2016) A20 [arXiv:1502.02114] [INSPIRE].

[29] B.-H. Lee and W. Lee, Vacuum bubbles in a de Sitter background and black hole pair creation, Class. Quant. Grav. 26 (2009) 225002 [arXiv:0809.4907] [INSPIRE].

[30] V. Balek and M. Demetrian, Euclidean action for vacuum decay in a de Sitter universe, Phys. Rev. D 71 (2005) 023512 [gr-qc/0409001] [INSPIRE].

[31] L.G. Jensen and P.J. Steinhardt, Bubble Nucleation and the Coleman-Weinberg Model, Nucl. Phys. B 237 (1984) 176 [INSPIRE].

[32] J.C. Hackworth and E.J. Weinberg, Oscillating bounce solutions and vacuum tunneling in de Sitter spacetime, Phys. Rev. D 71 (2005) 044014 [hep-th/0410142] [INSPIRE]. 
[33] J. Elias-Miro, J.R. Espinosa, G.F. Giudice, G. Isidori, A. Riotto and A. Strumia, Higgs mass implications on the stability of the electroweak vacuum, Phys. Lett. B 709 (2012) 222 [arXiv:1112.3022] [INSPIRE].

[34] D. Buttazzo et al., Investigating the near-criticality of the Higgs boson, JHEP 12 (2013) 089 [arXiv: 1307.3536] [INSPIRE].

[35] G. Isidori, G. Ridolfi and A. Strumia, On the metastability of the standard model vacuum, Nucl. Phys. B 609 (2001) 387 [hep-ph/0104016] [inSPIRE].

[36] B.-H. Lee, W. Lee, C. Oh, D. Ro and D.-h. Yeom, Fubini instantons in curved space, JHEP 06 (2013) 003 [arXiv: 1204.1521] [INSPIRE].

[37] B.-H. Lee, W. Lee, D. Ro and D.-h. Yeom, Oscillating Fubini instantons in curved space, Phys. Rev. D 91 (2015) 124044 [arXiv:1409.3935] [INSPIRE]. 